\title{
Dynamic Capabilities for Opportunity Exploration: Insights from an Explorative Case Study
}

\author{
Sandra Baumbach \\ FIM Research Center, University of Augsburg, \\ Universitätsstrasse 12, 86159 Augsburg, Germany \\ +496652-6575 \\ sandra.baumbach@t-online.de
}

\section{Maximilian Röglinger}

FIM Research Center, University of Bayreuth, Project Group Business \& Information Systems Engineering of the Fraunhofer FIT, Wittelsbacherring 10, 95447 Bayreuth, Germany +49 $92155-4710$

maximilian.roeglinger@fim-rc.de

\author{
Anna Maria Oberländer \\ FIM Research Center, \\ University of Bayreuth, \\ Wittelsbacherring 10, 95447 Bayreuth, \\ Germany \\ +4992155-4710 \\ anna.oberlaender@fim-rc.de
}

\section{Michael Rosemann}

Queensland University of Technology, 2 George Street, Brisbane Qld 4000, Australia

$+6173138-9473$

m.rosemann@qut.edu.au

Sandra Baumbach is a student of the Elite Graduate Program Finance and Information Management (M.Sc.) offered by the University of Augsburg and Technical University Munich. She worked as research assistant at the Research Center Finance \& Information Management (FIM) with a focus on digitization, opportunity management, and dynamic capabilities.

Anna Maria Oberländer is a research associate at the Research Center Finance \& Information Management with a focus on digitalization, digital innovation, and the (Industrial) Internet of Things. Anna is pursuing her $\mathrm{PhD}$ as part of an educational leave from McKinsey \& Company where she has worked as a senior consultant in the advanced industries and financial services sector.

Maximilian Röglinger is Professor of Information Systems and Value-based Business Process Management at the University of Bayreuth. Maximilian also serves as Deputy Academic Director of the Research Center Finance \& Information Management (FIM) and works in a leading position with the Project Group Business \& Information Systems Engineering of the Fraunhofer FIT. Maximilian's activities in research, teaching, and industry center around business process management, digital innovation, and digital production.

Michael Rosemann is the Director of the Centre for Future Enterprise and a Professor for Innovation Systems at Queensland University of Technology. His areas of interest are revenue resilience, innovation (eco)systems, systemic ideation, process management and trust management. As Head of QUT's Information Systems School he established the Woolworths Chair in Retail Innovation, the Brisbane Airport Chair in Airport Innovation and the PwC Chair in Digital Economy. Dr Rosemann is the author/editor of seven books, more than 300 refereed papers (incl. MISQ, EJIS, JAIS, IS), Editorial Board member of ten international journals and co-inventor of US patents. His publications have been translated into Russian, Mandarin, German and Portuguese. Michael is also the Honorary Consul for the Federal Republic of Germany in Brisbane since 2016. 


\begin{abstract}
Digital technologies offer organizations new opportunities. However, unlike well-defined problem-response strategies (e.g., Lean Management), it remains elusive how to identify and leverage opportunities, particularly for public sector organizations. As extant knowledge lacks corresponding theory-guided and empirically validated opportunity management practices, this study provides insights on opportunity management practices through an exploratory case study. The case of interest is Queensland Urban Utilities, a utility provider which developed a strong focus on opportunity exploration despite operating in a low-competition environment. Building upon organizational ambidexterity and dynamic capabilities as theoretical lenses, we present a conceptual framework distinguishing two opportunity types, namely core and new business opportunities. Along this framework, we present 15 practice areas and actionable practices, supported by real-life examples. Thereby, we identify two facets of exploration demanding divergent capabilities to sense and seize opportunities. Our study contributes to the understanding of exploration capabilities and supports practitioners in developing opportunity management practices.
\end{abstract}

\title{
Keywords:
}

Opportunity, opportunity management, organizational ambidexterity, opportunity exploration, dynamic capabilities, case study research, single case study 